Research Article

\title{
Identification of a Prognostic Risk Signature of Kidney Renal Clear Cell Carcinoma Based on Regulating the Immune Response Pathway Exploration
}

\author{
Guangzhen $W u\left(\mathbb{D},{ }^{1}\right.$ Yingkun Xu $\mathbb{D}^{2},{ }^{2}$ Chenglin Han $\mathbb{D}^{2},{ }^{2}$ Zilong Wang $\mathbb{D}^{2},{ }^{2} \mathrm{Jiayi}_{\mathrm{Li}}{ }^{3}$ \\ Qifei Wang $\left(\mathbb{1},{ }^{1}\right.$ and Xiangyu Che $\mathbb{1}^{1}$ \\ ${ }^{1}$ Department of Urology, The First Affiliated Hospital of Dalian Medical University, Dalian, China \\ ${ }^{2}$ Department of Urology, Shandong Provincial Hospital, Cheeloo College of Medicine, Shandong University, Jinan, China \\ ${ }^{3}$ School of Business, Hanyang University, Seoul, Republic of Korea
}

Correspondence should be addressed to Qifei Wang; wangqifei6008@hotmail.com and Xiangyu Che; dalianchexiangyu@163.com

Received 15 October 2020; Accepted 12 December 2020; Published 30 December 2020

Academic Editor: Yue Zhang

Copyright (C) 2020 Guangzhen Wu et al. This is an open access article distributed under the Creative Commons Attribution License, which permits unrestricted use, distribution, and reproduction in any medium, provided the original work is properly cited.

Purpose. To construct a survival model for predicting the prognosis of patients with kidney renal clear cell carcinoma (KIRC) based on gene expression related to immune response regulation. Materials and Methods. KIRC mRNA sequencing data and patient clinical data were downloaded from the TCGA database. The pathways and genes involved in the regulation of the immune response were identified from the GSEA database. A single factor Cox analysis was used to determine the association of mRNA in relation to patient prognosis $(P<0.05)$. The prognostic risk model was further established using the LASSO regression curve. The survival prognosis model was constructed, and the sensitivity and specificity of the model were evaluated using the ROC curve. Results. Compared with normal kidney tissues, there were 28 dysregulated mRNA expressions in KIRC tissues $(P<0.05)$. Univariate Cox regression analysis revealed that 12 mRNAs were related to the prognosis of patients with renal cell carcinoma. The LASSO regression curve drew a risk signature consisting of six genes: TRAF6, FYN, IKBKG, LAT2, C2, IL4, EREG, TRAF2, and IL12A. The five-year ROC area analysis (AUC) showed that the model has good sensitivity and specificity (AUC $>0.712$ ). Conclusion. We constructed a risk prediction model based on the regulated immune response-related genes, which can effectively predict the survival of patients with KIRC.

\section{Introduction}

Worldwide, renal cell carcinoma (RCC) is a common urinary system tumor, accounting for approximately $90 \%$ of all malignant renal tumors. Kidney renal clear cell carcinoma (KIRC) is the most common pathological subtype, accounting for approximately $75 \%$ of all RCCs [1]. In 2018, approximately 65,340 patients with kidney cancer were diagnosed in the United States alone, and nearly, 14,970 patients died from kidney cancer [2]. The latest statistics show that the incidence of KIRC is increasing at an annual rate of $2 \%$ [3]. Presently, patients with early KIRC mainly rely on surgical treatment. In the early stages of the disease, most patients do not present specific symptoms; thus, approximately one-third of patients have metastatic disease at the time of diagnosis. Patients with metastases and relapses lose the opportunity for radical surgery and instead undergo traditional radiotherapy and chemotherapy [4]. Although some molecularly targeted drugs for KIRC have been used, most patients eventually develop drug resistance [5]. Therefore, mining new target biomarkers related to KIRC diagnosis and treatment is a hot topic in current cancer research and an urgent task.

In recent years, the relationship of the immune system in the development and progression of cancer has become critical for research. The interaction between the immune 
system and the tumor can be divided into three stages: immune elimination, immune hold-up, and immune escape [6]. In the immune elimination stage, the body endogenously suppresses tumor cells through gene repair, aging, and apoptosis. Simultaneously, the danger signals produced by apoptotic cells and the antigens produced by tumor cells stimulate the immune system; thus, innate and adaptive immunity work together to create an antitumor effect. After the immune elimination phase, the remaining mutating tumor cells interact with the adaptive immune cells and pass through the immune phase. At this time, the growth of tumor cells is prevented by immune mechanisms. When the balance between tumor cells and the immune system is disrupted, however, tumor cells generate immune escape environments by producing immunosuppressive cytokines, such as VEGF and TGF- $\beta$, and recruiting regulatory immune cells with immunosuppressive effects, such as TREG and MDSC [7]. As one of the biological characteristics of tumors, the immune escape phase shows the dual role of the immune system in tumor occurrence and development.

While the tumor-infiltrating immune cells can effectively control tumor cell occurrence and development and eventually eliminate tumor cells, they can also help the tumor avoid immune attack and promote tumor growth $[8,9]$. In addition, in the process of tumor generation, exogenous pathways mediated by innate immune cells and endogenous pathways mediated by tumor cells work together to produce tumor-promoting inflammation in the tumor microenvironment, prompting tumors to break through the immune defense line [10]. Thus, tumor immunotherapy has become an important approach to tumor treatment.

In this study, we focused mainly on the potential role of genes related to immune response regulation in KIRC and successfully used these genes to establish a prognostic risk signature for patients with KIRC.

\section{Materials and Methods}

2.1. Data Collection. In June 2020, we downloaded the mRNA sequencing data of 539 cases of KIRC tissue and 72 cases of normal renal tissue from the TCGA database website with the clinicopathological data of patients (https://portal. gdc.cancer.gov/). The clinical information included age, sex, pathological stage, histological grade, survival time, and survival status. The pathway of immune response regulation was taken from the GSEA website (https://www.gseamsigdb.org/gsea/index.jsp). We collected 33 genes related to this pathway for this study.

\subsection{Construction of the PPI Network. The STRING database} (http://www.string-db.org) collects and integrates known and predicted protein-protein association data for many organisms, including humans [11]. In this study, the STRING database was used to predict the interaction between proteins encoded by the related genes regulating the immune response. The protein-protein interaction (PPI) was constructed on the condition that the interaction combination score was greater than 0.4. Unlinked protein molecules were removed, and the interaction data and corresponding images on the remaining protein molecules were arranged.

2.3. Data Processing and Analysis. Our data processing and statistics were implemented using multiple extension packages based on the $R$ language. First, we drew a heat map reflecting the expression of genes that regulate the immune response in KIRC. The "Pheatmap" extension package was used to draw heat maps, while the "limma" extension package was used to analyze mRNA differences. Then, we conducted a single factor COX analysis of these molecules in KIRC to show the relationship between these molecules and the prognosis of patients with kidney cancer. Next, we used the "corrplot" extension software package to map the coexpression relationship between genes involved in regulating the immune response. The "glmnet" and "survival" extension software packages were used to draw the LASSO regression curve and survival curve. The risk score was calculated based on a linear combination of the Cox coefficient and gene expression. The following calculation formula was used for the analysis: risk score $=\sum^{N}{ }_{i=1}$ (Expi*Coei). N, Coei, and Expi represent the gene number, coefficient value, and level of gene expression, respectively. The median was used to divide the patients into low-risk and high-risk groups. The corresponding survival curve was drawn. To verify the accuracy of the model, we used the "survival ROC" expansion package to obtain five-year and ten-year ROC curves. Finally, based on this model, we analyzed the correlation with the pathological features of RCC seen in patients and displayed the correlation as a heat map.

2.4. TIMER Database Analysis. TIMER is a database for the systematic analysis of various types of tumor immune information (https://cistrome.shinyapps.io/timer/) [12, 13]. The TIMER database contains data from 10897 samples of 32 cancers from TCGA and can assess the abundance of tumor immune invasion. The "Gene" module was used to analyze the correlation between the expression level of the target gene in KIRC and the infiltration abundance of immune cells. In the TIMER database, we analyzed the correlation between the genes that construct risk signatures and tumor immune infiltrating cells and used the Spearman correlation coefficient to express their relationship.

\section{Results}

3.1. The Expression of Regulated Immune Response-Related Genes in KIRC and the Interaction between Various Molecules. A flow chart displaying the overall design and analysis process of this research is presented in Figure 1. To explore the expression of genes related to the immune response regulation in clear cell RCC, we combined the $R$ language with the mRNA expression data from the KIRC dataset in the TCGA database to draw a heat map of these genes. Twenty-eight of 33 genes were differentially expressed in KIRC tissues and normal kidney tissues (Figure 2(a)). Thus, 


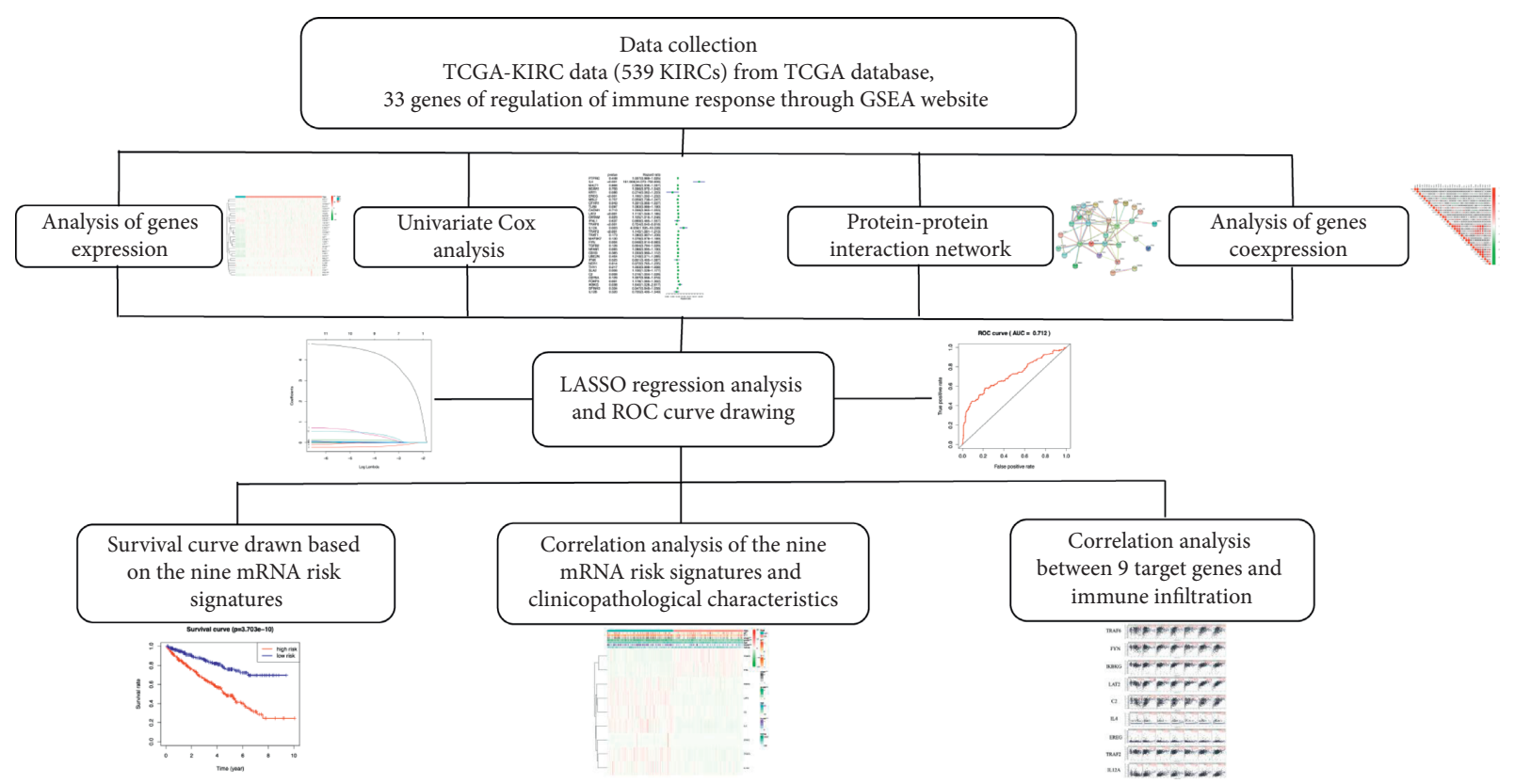

FIgUre 1: The flow chart of the study design and analysis.

the pathway regulating the immune response plays an important role in the development of clear cell RCC. The univariate COX analysis revealed ten molecules, IL4, EREG, LAT2, CRTAM, IL12A, TRAF2, SLA2, C2, FOXP3, and IKBKG, that are risk factors for the occurrence and development of KIRC. TRAF6 and FYN play a protective role in the occurrence and development of KIRC (Figure 2(b)). The interactions between various molecules are displayed in the PPI network (Figure 2(c)). The subsequent coexpression analysis revealed a strong coexpression relationship between MBL2 and CFHR1 (Figure 2(d)).

3.2. Construction and Verification of Risk Signatures. To establish risk signatures in KIRC using genes related to the regulation of the immune response, we used lasso regression analysis to select a model consisting of nine genes (Figure 3(a)) and cross-validated the feasibility of this model (Figure 3(b)). Using this model, patients with KIRC can be divided into high-risk and low-risk groups. The overall survival rate of patients in the high-risk group was significantly lower than that of patients in the low-risk group $(P=$ $3.703 e-10)$ (Figure 3(c)). R3.6.0 software was used to draw the ROC curve of the model to evaluate the model's sensitivity and specificity. The model predicts the area under the ROC curve (AUC) of the patients' 5-year survival rate (Figure 3(d)) and 10-year survival rate (Figure 3(e)). These rates were 0.712 and 0.752 , respectively, indicating that the model has good sensitivity and specificity. These results suggest that the prognostic model we constructed can predict the survival of patients with clear cell RCC. When we combined this model with clinicopathological features, we found that this model was correlated with $M, T$, stage, grade, and fustat parameters (Figure 3(f)).
3.3. The Relationship between Regulated Immune ResponseRelated Genes and Various Immune Cells. The results showed that there were correlations between the expressions of IKBKG, LAT2, and C2 and the infiltration of purity, B cells, CD8+ T cells, CD4+ T cells, macrophages, neutrophils, and dendritic cells. In addition, there was a correlation between the expressions of TRAF6 and IL12A and the infiltration of B cells, CD8+ T cells, CD4+ T cells, macrophages, neutrophils, and dendritic cells. There was a correlation between the expression of FYN molecules and the infiltration of purity, CD8+ T cells, CD4+ T cells, macrophages, neutrophils, and dendritic cells. Furthermore, there was a correlation between the expression of TRAF2 and the infiltration of B cells, CD8+ T cells, CD4+ $\mathrm{T}$ cells, neutrophils, and dendritic cells. There was a correlation between EREG expression and the infiltration of purity, CD4+ T cells, macrophages, and neutrophils (Figure 4).

\section{Discussion}

Bioinformatics analysis methods have been increasingly used in the medical field $[14,15]$. In this study, we used various bioinformatics methods to deeply study the potential role of pathways regulating immune responses in KIRC. The KIRC prognostic model illustrates the relationship between the pathway regulating immune responses and the occurrence and development of KIRC. The prognostic model can effectively predict the overall survival rate of patients with KIRC. The Timer Online website suggests that these nine target genes are related to the infiltration of different immune cell types. This discovery provides a new strategy for the study of KIRC prognosis from the perspective of immune cell infiltration. 


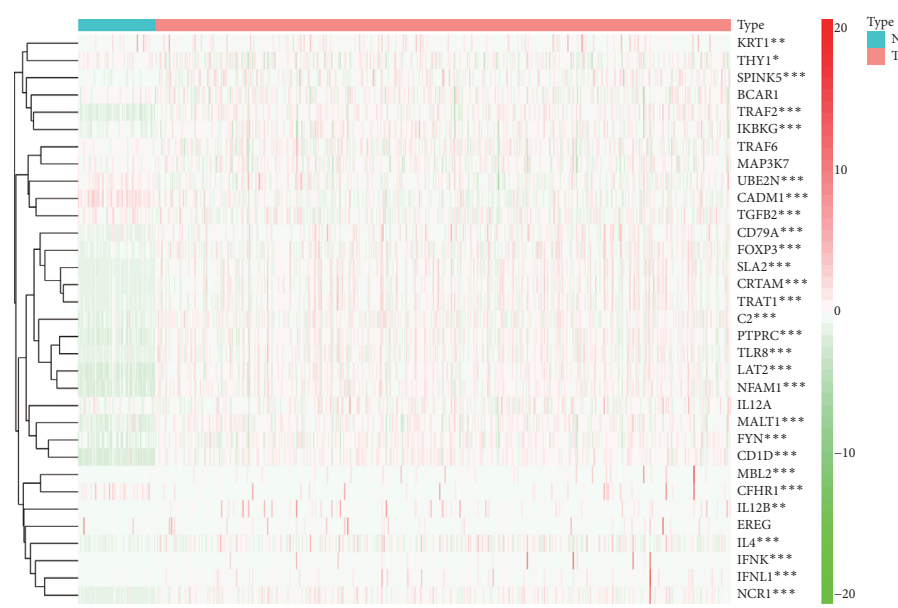

(a)

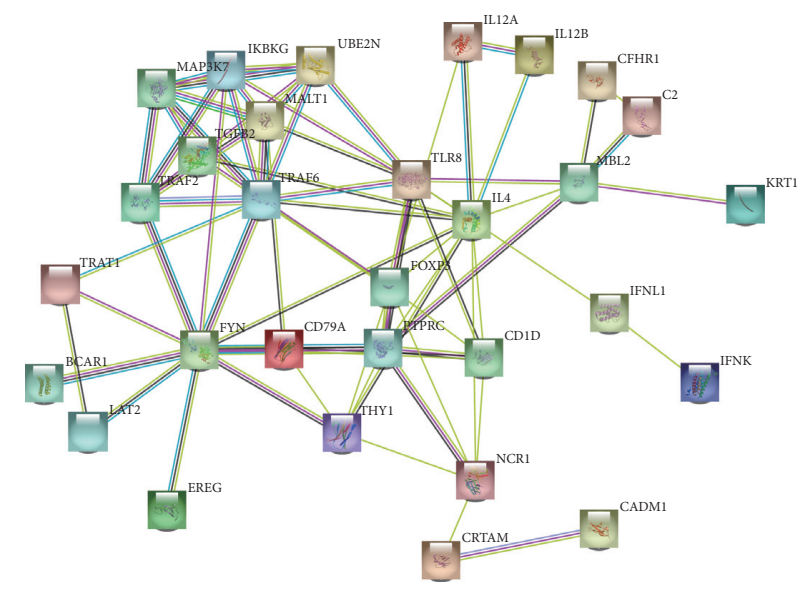

(c)



(b)

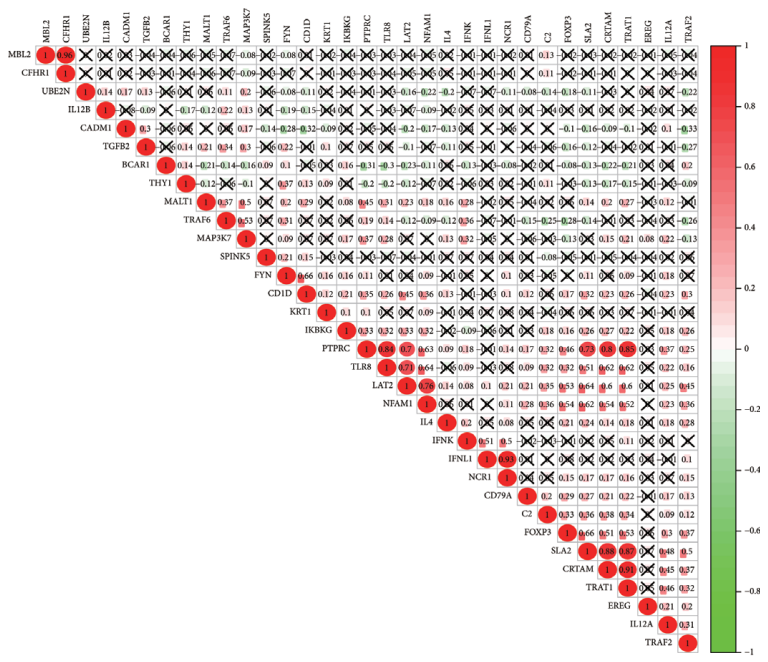

(d)

Figure 2: (a) A heat map showing the expression of genes that regulate the immune response between renal clear cell carcinoma and normal tissues. (b) A heat map showing the hazard ratio analysis of genes related to immune response regulation in KIRC. (c) The PPI network between immune response-related molecules. (d) Coexpression between immune-related molecules. ${ }^{*} P<0.05 ;{ }^{* *} P<0.01$; ${ }^{* * *} P<0.001$.

TRAF6, from the TRAF family, is involved in the transduction of inflammatory signals $[16,17]$. Studies have shown that it can regulate tumorigenesis and angiogenesis in lung and pancreatic cancers [18-20]. In addition, it has been reported that TRAF6 inhibits the transfer of colorectal cancer by regulating autophagy [21]. Chen et al. confirmed that the activation of the FYN protein promotes the phosphorylation of Sam68, protecting pancreatic cancer cells from apoptosis and promoting the proliferation and metastasis of pancreatic cancer cells [22]. The FYN protein plays a role in promoting cancer by regulating the translation and activity of COX2 in prostate cancer [23]. In addition, FYN protein induces EMT in breast cancer, promoting tumor cell invasion and metastasis [24]. The FYN protein is also associated with breast cancer chemotherapy drug sensitivity [25]. A previous study showed that IKBKG in KIRC could prevent cancer cells from entering apoptosis by regulating HIF, promoting cancer cell survival and tumor metastasis [26]. LAT2 is overexpressed and plays a carcinogenic role in pancreatic cancer. It can also reduce the sensitivity of gemcitabine by modulating the LAT2-mTORLDHB signaling pathway [27]. Existing research shows that $\mathrm{C} 2$ gene polymorphism is closely related to chronic hepatitis $B$, hepatitis B virus-related cirrhosis, and liver cancer [28]. Chang et al. found that the high IL4 expression in KIRC is associated with increased tumor recurrence and decreased patient survival. In KIRC, the parameter IL4/IL13 is an independent prognostic factor for RFS and OS $[29,30]$. EREG plays a carcinogenic role by binding to EGFR in various human tumors, such as colorectal cancer [31,32], nonsmall cell lung cancer [33-35], and gliomas [36]. TRAF2 is similar to TRAF6 and plays an important role in inflammation, immune response, and malignant tumor infiltration [37-40]. Similarly, our results show that both TRAF2 and TRAF6 are significantly related to B cells, CD8+ T cells, CD4+ T cells, neutrophils, and dendritic cells. This shows that there is a strong correlation between TRAF2 and TRAF6 and immune infiltration. In addition, TRAF2 is 


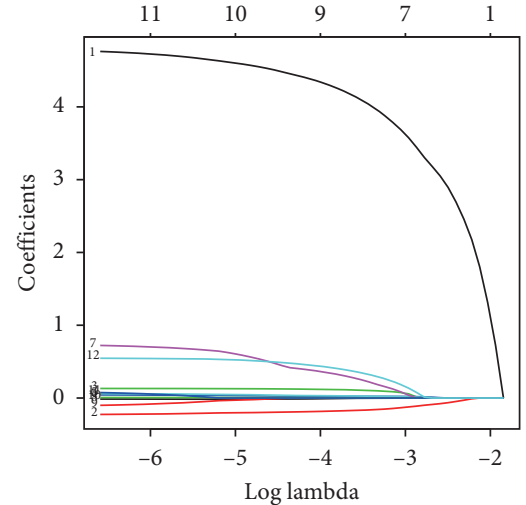

(a)

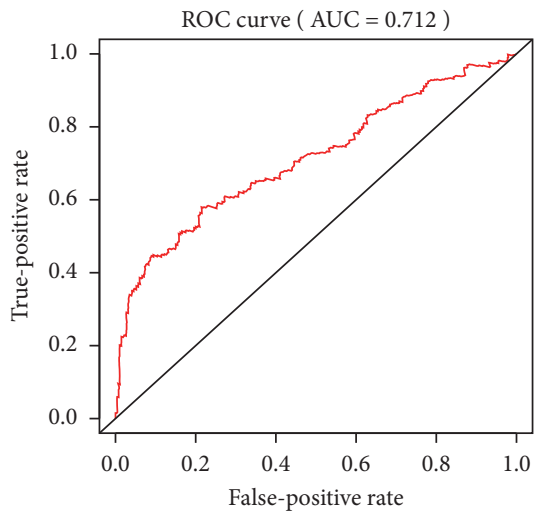

(d)

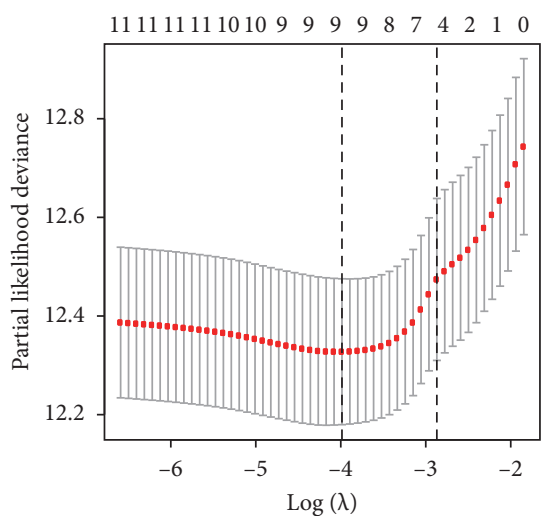

(b)

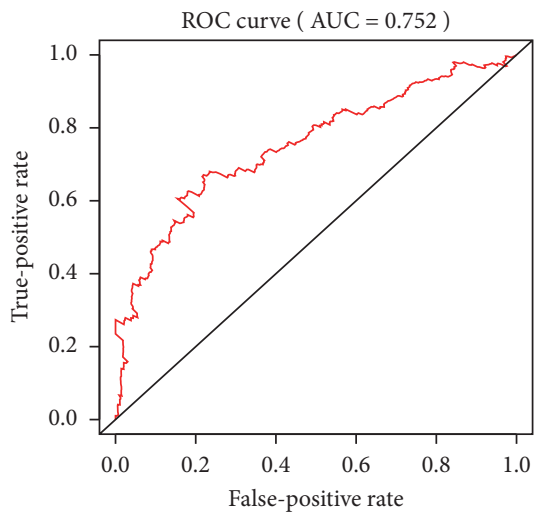

(e)

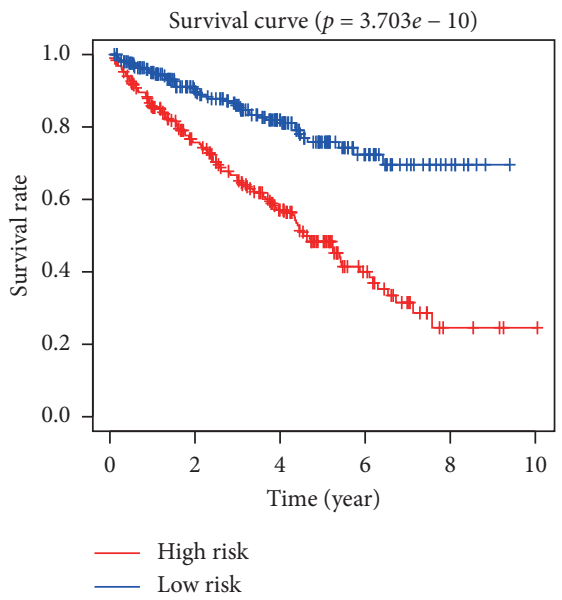

(c)

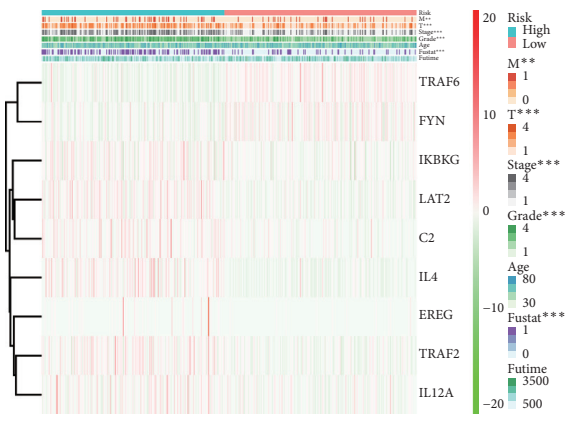

(f)

FIGURE 3: (a)-(b) Establishment of a risk signature using the LASSO regression curve and verification. (c) The survival curve analysis in KIRC based on the risk model. (d) Five-year ROC curve. (e) Ten-year ROC curve. (f) The relationship with clinicopathological features based on this model. ${ }^{* *} P<0.01 ;{ }^{* * *} P<0.001$.

upregulated in breast cancer, pancreatic cancer, and other malignant tumors $[41,42]$ and is significantly related to the prognosis of patients with prostate cancer [43]. Zhang et al. found that IL12A showed significantly high expression in differentiated thyroid cancer, was associated with disease invasiveness, and was an independent predictor of the prognosis of differentiated thyroid cancer [44].

The above indicates that these target genes utilize different molecular mechanisms in participating in the occurrence and development of various human malignant tumors. These target genes can promote malignant transformation in some tissues and inhibit tumorigenesis in other tissues. The abnormal expression of these molecules is closely related to the clinicopathological characteristics of the tumor and the prognosis of patients. In the future, these genes can become potential biological targets for tumor prevention, diagnosis, and treatment. Among them, studies on the potential functions of TRAF6, FYN, LAT2, and other molecules in KIRC are still relatively few. Therefore, the specific molecular mechanism of how these molecules affect tumorigenesis and development in the future is worthy of indepth study.

Although this study has made progress in the prognosis of KIRC, there are some limitations. The study used public datasets, making it impossible to obtain all the information needed by each patient. This entails that some patients with acute infections, diseases of the immune system, or using anti-inflammatory drugs may have been included in this study. Ideally, these patients should be excluded. In addition, the gene expression data of normal kidney tissue are less than that of tumor tissue in the currently available tumor genome atlas database, which lead to an imbalance in the sample when collecting the data. However, with the development of current high-throughput gene expression detection technology, more and more gene expression data will be available for investigation. In future studies, we will collect more data on patients with KIRC and normal tissue data and continue to conduct further research. 

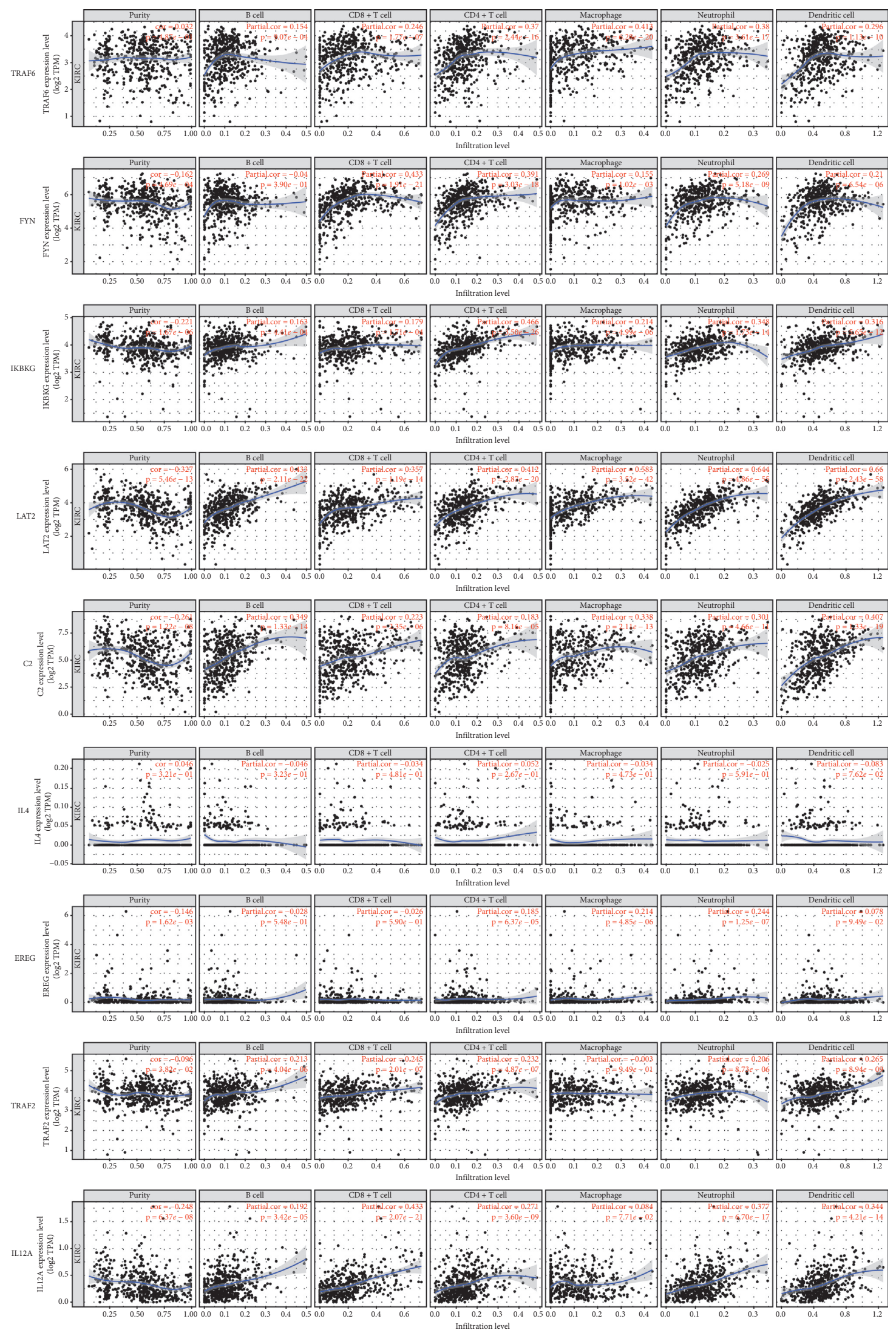

Figure 4: Correlation between TRAF6, FYN, IKBKG, LAT2, C2, IL4, EREG, TRAF2, IL12A, and various immune cells. 


\section{Conclusion}

In summary, we constructed a risk model for the survival of patients with clear cell renal cell carcinoma composed of 9 genes that modulate the immune response. The model has good sensitivity and specificity and indicates that patients in the high-risk group have a greater risk of death. The study showed that the establishment of multigene prognostic models can provide accurate prognostic guidance and has an important reference value for the selection of individualized treatment plans. The risk model can effectively predict the survival of patients with permeable clear cell renal cell carcinoma. Even so, the model still needs to be further verified using large-scale multicenter evidence-based medical evidence.

\section{Abbreviations}

KIRC: Kidney renal clear cell carcinoma

TCGA: The cancer genome atlas

GSEA: Gene set enrichment analysis

TRAF6: TNF receptor associated factor 6

FYN: $\quad$ FYN protooncogene

IKBKG: Inhibitor of nuclear factor kappa B kinase regulatory subunit gamma

LAT2: Linker for activation of T cells family member 2

C2: $\quad$ Complement C2

IL4: Interleukin 4

EREG: Epiregulin

TRAF2: TNF receptor associated factor 2

IL12A: Interleukin 12A

RCC: Renal cell carcinoma

TREG: Regulatory T cell

MDSC: Myeloid-derived suppressor cell.

\section{Data Availability}

The data used to support the findings of this study are available from the corresponding author upon request.

\section{Disclosure}

Guangzhen $\mathrm{Wu}$ and Yingkun $\mathrm{Xu}$ are the co-first authors.

\section{Conflicts of Interest}

The authors declare that they have no conflicts of interest.

\section{Authors' Contributions}

Guangzhen $\mathrm{Wu}$ and Yingkun Xu contributed equally to this study. Qifei Wang and Xiangyu Che designed the study; Guangzhen $\mathrm{Wu}$ and Yingkun $\mathrm{Xu}$ searched and extracted data; Zilong Wang, Chenglin Han, and Jiayi Li analyzed the data; Yingkun Xu finished writing the manuscript. All authors reviewed the manuscript and approved the submitted version.

\section{Acknowledgments}

The authors thank the TCGA database for providing a lot of reliable data. This project was supported by the Liaoning Provincial Department of Education's 2020 scientific research project (No. LZ2020071).

\section{References}

[1] R. L. Siegel, K. D. Miller, and A. Jemal, "Cancer statistics, 2020," CA: A Cancer Journal for Clinicians, vol. 70, no. 1, pp. 7-30, 2020.

[2] R. L. Siegel, K. D. Miller, and A. Jemal, "Cancer statistics, 2018," CA: A Cancer Journal for Clinicians, vol. 68, no. 1, pp. 7-30, 2018.

[3] D. J. Grignon and M. Che, "Clear cell renal cell carcinoma," Clinics in Laboratory Medicine, vol. 25, no. 2, pp. 305-316, 2005.

[4] F. E. Vera-Badillo, A. J. Templeton, I. Duran et al., "Systemic therapy for non-clear cell renal cell carcinomas: a systematic review and meta-analysis," European Urology, vol. 67, no. 4, pp. 740-749, 2015.

[5] P. C. Barata and B. I. Rini, "Treatment of renal cell carcinoma: current status and future directions," CA: A Cancer Journal for Clinicians, vol. 67, no. 6, pp. 507-524, 2017.

[6] R. D. Schreiber, L. J. Old, and M. J. Smyth, "Cancer immunoediting: integrating immunity's roles in cancer suppression and promotion," Science, vol. 331, no. 6024, pp. 1565-1570, 2011.

[7] A. Sica and M. Massarotti, "Myeloid suppressor cells in cancer and autoimmunity," Journal of Autoimmunity, vol. 85, pp. 117-125, 2017.

[8] S. Vasudevan, Y. Tong, and J. A. Steitz, "Switching from repression to activation: microRNAs can up-regulate translation," Science, vol. 318, no. 5858, pp. 1931-1934, 2007.

[9] M. A. Cortez, S. Anfossi, R. Ramapriyan et al., "Role of miRNAs in immune responses and immunotherapy in cancer," Genes, Chromosomes and Cancer, vol. 58, no. 4, pp. 244-253, 2019.

[10] E. Allen, I. B. Walters, and D. Hanahan, "Brivanib, a dual FGF/VEGF inhibitor, is active both first and second line against mouse pancreatic neuroendocrine tumors developing adaptive/evasive resistance to VEGF inhibition," Clinical Cancer Research, vol. 17, no. 16, pp. 5299-5310, 2011.

[11] D. Szklarczyk, A. L. Gable, D. Lyon et al., "STRING v11: protein-protein association networks with increased coverage, supporting functional discovery in genome-wide experimental datasets," Nucleic Acids Research, vol. 47, no. D1, pp. D607-d613, 2019.

[12] T. Li, J. Fu, Z. Zeng et al., "TIMER2.0 for analysis of tumorinfiltrating immune cells," Nucleic Acids Research, vol. 48, no. W1, pp. W509-W514, 2020.

[13] T. Li, J. Fan, B. Wang et al., "TIMER: a web server for comprehensive analysis of tumor-infiltrating immune cells," Cancer Research, vol. 77, no. 21, pp. e108-e110, 2017.

[14] Y. Xu, G. Wu, J. Li et al., "Screening and identification of key biomarkers for bladder cancer: a study based on TCGA and GEO data," Biomed Research International, vol. 2020, Article ID 8283401, 20 pages, 2020.

[15] Y. Xu, X. Li, Y. Han et al., "A new prognostic risk model based on PPAR pathway-related genes in kidney renal clear cell carcinoma," PPAR Research, vol. 2020, Article ID 6937475, 13 pages, 2020. 
[16] T. Ishida, S.-i. Mizushima, S. Azuma et al., "Identification of TRAF6, a novel tumor necrosis factor receptor-associated factor protein that mediates signaling from an amino-terminal domain of the CD40 cytoplasmic region," Journal of Biological Chemistry, vol. 271, no. 46, pp. 28745-28748, 1996.

[17] Z. Cao, J. Xiong, M. Takeuchi, T. Kurama, and D. V. Goeddel, "TRAF6 is a signal transducer for interleukin-1," Nature, vol. 383, no. 6599, pp. 443-446, 1996.

[18] D. T. Starczynowski, W. W. Lockwood, S. Deléhouzée et al., "TRAF6 is an amplified oncogene bridging the RAS and NF$\kappa \mathrm{B}$ pathways in human lung cancer," Journal of Clinical Investigation, vol. 121, no. 10, pp. 4095-4105, 2011.

[19] Y. Rong, D. Wang, W. Wu et al., "TRAF6 is over-expressed in pancreatic cancer and promotes the tumorigenicity of pancreatic cancer cells," Medical Oncology, vol. 31, no. 11, p. 260, 2014.

[20] H. Sun, X.-B. Li, Y. Meng, L. Fan, M. Li, and J. Fang, “TRAF6 upregulates expression of HIF-1 and promotes tumor angiogenesis," Cancer Research, vol. 73, no. 15, pp. 4950-4959, 2013.

[21] H. Wu, X.-X. Lu, J.-R. Wang et al., “TRAF6 inhibits colorectal cancer metastasis through regulating selective autophagic CTNNB1/ $\beta$-catenin degradation and is targeted for GSK3B/ GSK3 $\beta$-mediated phosphorylation and degradation," Autophagy, vol. 15, no. 9, pp. 1506-1522, 2019.

[22] Z.-Y. Chen, L. Cai, J. Zhu et al., "Fyn requires HnRNPA2B1 and Sam68 to synergistically regulate apoptosis in pancreatic cancer," Carcinogenesis, vol. 32, no. 10, pp. 1419-1426, 2011.

[23] A. Alexanian, B. Miller, M. Chesnik, S. Mirza, and A. Sorokin, "Post-translational regulation of COX2 activity by FYN in prostate cancer cells," Oncotarget, vol. 5, no. 12, pp. 4232-4243, 2014.

[24] Y.-G. Xie, Y. Yu, L.-K. Hou, X. Wang, B. Zhang, and X.-C. Cao, "FYN promotes breast cancer progression through epithelial-mesenchymal transition," Oncology Reports, vol. 36, no. 2, pp. 1000-1006, 2016.

[25] D. Elias, H. Vever, A.-V. Lænkholm et al., "Gene expression profiling identifies FYN as an important molecule in tamoxifen resistance and a predictor of early recurrence in patients treated with endocrine therapy," Oncogene, vol. 34, no. 15, pp. 1919-1927, 2015.

[26] A. M. Nowicka, I. Häuselmann, L. Borsig et al., "A novel pVHL-independent but NEMO-driven pathway in renal cancer promotes HIF stabilization," Oncogene, vol. 35 , no. 24 , pp. 3125-3138, 2016.

[27] M. Feng, G. Xiong, Z. Cao et al., "LAT2 regulates glutaminedependent mTOR activation to promote glycolysis and chemoresistance in pancreatic cancer," Journal of Experimental and Clinical Cancer Research, vol. 37, no. 1, p. 274, 2018.

[28] G. Ning, L. M. Zhen, W. X. Xu et al., "Suppression of complement component 2 expression by hepatitis B virus contributes to the viral persistence in chronic hepatitis B patients," Journal of Viral Hepatitis, vol. 27, no. 10, pp. 1071-1081, 2020.

[29] Y. Chang, L. Xu, H. An et al., "Expression of IL-4 and IL-13 predicts recurrence and survival in localized clear-cell renal cell carcinoma," International Journal of Clinical and Experimental Pathology, vol. 8, no. 2, pp. 1594-1603, 2015.

[30] J. T. Gibson, K. E. Norris, G. Wald et al., "Obesity induces limited changes to systemic and local immune profiles in treatment-naive human clear cell renal cell carcinoma," PLoS One, vol. 15, no. 5, p. e0233795, 2020.
[31] A. Lièvre, J.-B. Bachet, D. Le Corre et al., "KRAS mutation status is predictive of response to cetuximab therapy in colorectal cancer," Cancer Research, vol. 66, no. 8, pp. 3992-3995, 2006.

[32] S. Khambata-Ford, C. R. Garrett, N. J. Meropol et al., "Expression of epiregulin and amphiregulin and K-ras mutation status predict disease control in metastatic colorectal cancer patients treated with cetuximab," Journal of Clinical Oncology, vol. 25, no. 22, pp. 3230-3237, 2007.

[33] M. Takahashi, K. i. Hayashi, K. Yoshida et al., "Epiregulin as a major autocrine/paracrine factor released from ERK- and p38MAPK-activated vascular smooth muscle cells," Circulation, vol. 108, no. 20, pp. 2524-2529, 2003.

[34] M.-C. Cho, H.-S. Choi, S. Lee et al., "Epiregulin expression by Ets-1 and ERK signaling pathway in Ki-ras-transformed cells," Biochemical and Biophysical Research Communications, vol. 377, no. 3, pp. 832-837, 2008.

[35] J. Zhang, K. Iwanaga, K. C. Choi et al., "Intratumoral epiregulin is a marker of advanced disease in non-small cell lung cancer patients and confers invasive properties on EGFRmutant cells," Cancer Prevention Research, vol. 1, no. 3, pp. 201-207, 2008.

[36] P. H. Huang, A. M. Xu, and F. M. White, "Oncogenic EGFR signaling networks in glioma," Science Signaling, vol. 2, no. 87, p. re6, 2009.

[37] A. Borghi, L. Verstrepen, and R. Beyaert, "TRAF2 multitasking in TNF receptor-induced signaling to NF- $\kappa \mathrm{B}, \mathrm{MAP}$ kinases and cell death," Biochemical Pharmacology, vol. 116, pp. 1-10, 2016.

[38] X. Wu, R. Xue, H. Peng et al., "Traf6 inhibitor boosts antitumor immunity by impeding regulatory $\mathrm{T}$ cell migration in Hepa1-6 tumor model," International Immunopharmacology, vol. 77, p. 105965, 2019.

[39] J. Fang, L. C. Bolanos, K. Choi et al., "Ubiquitination of hnRNPA1 by TRAF6 links chronic innate immune signaling with myelodysplasia," Nature Immunology, vol. 18, no. 2, pp. 236-245, 2017.

[40] N. Xiao, H. Li, J. Luo et al., "Ubiquitin-specific protease 4 (USP4) targets TRAF2 and TRAF6 for deubiquitination and inhibits TNF $\alpha$-induced cancer cell migration," Biochemical Journal, vol. 441, no. 3, pp. 979-987, 2012.

[41] Z.-J. Zhao, H.-Y. Ren, F. Yang, J. Wang, G.-P. Wu, and X.-Y. Mi, "Expression, correlation, and prognostic value of TRAF2 and TRAF4 expression in malignant plural effusion cells in human breast cancer," Diagnostic Cytopathology, vol. 43, no. 11, pp. 897-903, 2015.

[42] A. Trauzold, C. Röder, B. Sipos et al., "CD95 and TRAF2 promote invasiveness of pancreatic cancer cells," Faseb Journal, vol. 19, no. 6, pp. 620-622, 2005.

[43] B. Wei, J. Liang, J. Hu et al., "TRAF2 is a valuable prognostic biomarker in patients with prostate cancer," Medical Science Monitor, vol. 23, pp. 4192-4204, 2017.

[44] G.-Q. Zhang, C.-T. Shen, H.-J. Song, Z.-L. Qiu, and Q.-Y. Luo, "High expression of interleukin-12a and its association with the clinicopathology and prognosis of differentiated thyroid cancer," European Thyroid Journal, vol. 9, no. 3, pp. 139-147, 2020. 